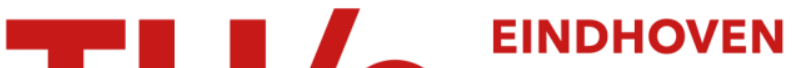

\section{New Methods for Computing the Terminal Cost for Min-max Model Predictive Control}

\section{Citation for published version (APA):}

Lazar, M., Munoz de la Pena, D., Heemels, W. P. M. H., \& Alamo, T. (2007). New Methods for Computing the Terminal Cost for Min-max Model Predictive Control. In Proceedings of the 2007 American Control Conference (ACC 2007) 9-13 July 2007, New York, New York, USA (pp. 4476-4481). Institute of Electrical and Electronics Engineers. https://doi.org/10.1109/ACC.2007.4282648

DOI:

10.1109/ACC. 2007.4282648

Document status and date:

Published: 01/01/2007

\section{Document Version:}

Publisher's PDF, also known as Version of Record (includes final page, issue and volume numbers)

\section{Please check the document version of this publication:}

- A submitted manuscript is the version of the article upon submission and before peer-review. There can be important differences between the submitted version and the official published version of record. People interested in the research are advised to contact the author for the final version of the publication, or visit the $\mathrm{DOI}$ to the publisher's website.

- The final author version and the galley proof are versions of the publication after peer review.

- The final published version features the final layout of the paper including the volume, issue and page numbers.

Link to publication

\section{General rights}

Copyright and moral rights for the publications made accessible in the public portal are retained by the authors and/or other copyright owners and it is a condition of accessing publications that users recognise and abide by the legal requirements associated with these rights.

- Users may download and print one copy of any publication from the public portal for the purpose of private study or research.

- You may not further distribute the material or use it for any profit-making activity or commercial gain

- You may freely distribute the URL identifying the publication in the public portal.

If the publication is distributed under the terms of Article 25fa of the Dutch Copyright Act, indicated by the "Taverne" license above, please follow below link for the End User Agreement:

www.tue.nl/taverne

Take down policy

If you believe that this document breaches copyright please contact us at:

openaccess@tue.nl

providing details and we will investigate your claim. 


\section{New Methods for Computing the Terminal Cost for Min-max Model Predictive Control}

M. Lazar, Member, IEEE
D. Muñoz de la Peña

\author{
W.P.M.H. Heemels
}

\begin{abstract}
The aim of this paper is to provide new techniques for computing a terminal cost and a local state-feedback control law that satisfy recently developed min-max MPC input-to-state stabilization conditions. Min-max MPC algorithms based on both quadratic and 1-norms or $\infty$-norms costs are considered. Compared to existing approaches, the proposed techniques can be applied to linear systems affected simultaneously by timevarying parametric uncertainties and additive disturbances. The resulting MPC cost function is continuous, convex and bounded, which is desirable from an optimization point of view. Regarding computational complexity aspects, the developed techniques employ linear matrix inequalities in the case of quadratic MPC cost functions and, norm inequalities in the case of MPC cost functions defined using 1-norms or $\infty$-norms. The effectiveness of the developed methods is illustrated for an active suspension application example.
\end{abstract}

Index Terms-Min-max, predictive control, stability.

\section{INTRODUCTION}

One of the practically relevant problems in control theory is the robust regulation towards a desired equilibrium of discrete-time systems affected, possibly simultaneously, by time-varying parametric uncertainties and additive disturbance inputs. In the case when hard constraints are imposed on state and input variables, the min-max model predictive control (MPC) methodology provides a reliable solution for tackling this control problem, see, for example, [1] for an overview. The research related to min-max MPC is focused on solving efficiently min-max MPC optimization problems on one hand, e.g., [2]-[4], and guaranteeing stability of the controlled system, on the other hand, e.g. [1], [5]-[7]. In this paper we are interested in stability issues and therefore, we focus our attention on results developed in this direction.

Most of the stability results obtained for perturbed discrete-time systems in closed-loop with min-max MPC controllers use a terminal cost and constraint set approach [1]. Although sufficient conditions that must be satisfied to guarantee stability of a min-max MPC closed-loop system are known [1], techniques to determine a suitable terminal cost and constraint set are non-trivial and of interest [4]-[8]. For linear systems affected only by parametric uncertainties,

Mircea Lazar is with the Department of Electrical Engineering and Maurice Heemels is with the Department of Mechanical Engineering, Eindhoven University of Technology, P.O. Box 513, 5600 MB Eindhoven, The Netherlands, E-mail: m.lazardtue.nl, m.heemels@tue.nl.

Daivd Muñoz de la Peña is with the Department of Chemical Engineering, University of California, Los Angeles, Los Angeles, CA 90095, USA, Email: davidmpseucla.edu.

Teodoro Alamo is with the Departamentos de Inginería de Sistemas y Automática, Universidad de Seville, 41092 Seville, Spain, E-mail: alamo@cartuja.us.es. linear matrix inequalities (LMI) based design techniques were derived in [9], [3]. However, these LMI stabilization conditions are not guaranteeing robustness (in terms of inputto-state stability [10]) when additive disturbance inputs affect the system. Ultimate boundedness of linear systems affected only by additive disturbance inputs in closed-loop with minmax MPC was guaranteed in [8] by setting the terminal cost equal to zero for all states and using a discontinuous stage cost, which is equal to zero for all states in the terminal set. In [4], an LMI technique for designing the terminal cost for a given linear feedback law was provided for systems subject to additive uncertainties.

In this paper we develop techniques for calculating both a non-zero terminal cost and a local state-feedback controller such that recently developed input-to-state stabilization conditions for min-max MPC [5]-[7] are satisfied. These techniques employ LMIs for quadratic MPC costs and norm inequalities for MPC costs defined using 1-norms or $\infty$-norms. Compared to the above-mentioned existing approaches, the proposed methods for computing a terminal cost can be applied to systems affected by both parametric uncertainties and additive disturbances. One of their advantages, from an optimization point of view, is that the resulting MPC cost function is continuous, convex and bounded. The computational methods presented in this paper can be employed to ensure input-to-state practical stability [10], [6] for various feedback or open-loop min-max MPC schemes, such as the ones in [2]-[4], [8], [9], [11], [12].

The paper is organized as follows. Section II introduces some preliminaries, regarding the nomenclature, the class of systems and min-max MPC stability notions. The problem statement is presented in Section III, while the techniques for computing a terminal cost and a local state-feedback control law are presented in Section IV. An active suspension application example illustrates the developed theory in Section V. Conclusions are summarized in Section VI.

\section{PRELIMINARIES}

\section{A. Nomenclature and basic definitions}

Let $\mathbb{R}, \mathbb{R}_{+}, \mathbb{Z}$ and $\mathbb{Z}_{+}$denote the field of real numbers, the set of non-negative reals, the set of integers and the set of non-negative integers, respectively. We use the notation $\mathbb{Z}_{\geq c}$ to denote the set $\left\{k \in \mathbb{Z}_{+} \mid k \geq c\right\}$ for some $c \in \mathbb{Z}_{+}$and $\mathbb{Z}^{N}$ to denote the $N$-times Cartesian product $\mathbb{Z} \times \ldots \times \mathbb{Z}$, for some $N \in \mathbb{Z}_{\geq 1}$. A polyhedron (or a polyhedral set) is a set obtained as the intersection of a finite number of open and/or closed half-spaces. A function $\sigma: \mathbb{R}_{+} \rightarrow \mathbb{R}_{+}$belongs to class $\mathscr{K}$ $(\sigma \in \mathscr{K})$ if it is continuous, strictly increasing and $\sigma(0)=0$. 
The Hölder $p$-norm of a vector $x \in \mathbb{R}^{n}$ is defined as:

$$
\|x\|_{p}:=\left\{\begin{array}{l}
\left(\left|x_{1}\right|^{p}+\ldots+\left|x_{n}\right|^{p}\right)^{\frac{1}{p}}, \quad p \in \mathbb{Z}_{[1, \infty)} \\
\max _{i=1, \ldots, n}\left|x_{i}\right|, \quad p=\infty
\end{array}\right.
$$

where $x_{i}, i=1, \ldots, n$ is the $i$-th component of $x$. For a matrix $Z \in \mathbb{R}^{m \times n}$ we define $\|Z\|_{p}:=\sup _{x \neq 0} \frac{\|Z x\|_{p}}{\|x\|_{p}}, p \in \mathbb{Z}_{\geq 1}$, as the induced matrix norm. It is known that $\|Z\|_{\infty}=$ $\max _{1 \leq i \leq m} \sum_{j=1}^{n}\left|Z^{\{i j\}}\right|$, where $Z^{\{i j\}}$ is the $i j$-th entry of $Z$. For a matrix $Z \in \mathbb{R}^{m \times n}$ with full-column rank, $Z^{-L}:=$ $\left(Z^{\top} Z\right)^{-1} Z^{\top}$ denotes its left Moore-Penrose inverse, which satisfies $Z^{-L} Z=I_{n}$. For a positive definite matrix $Z, Z^{\frac{1}{2}}$ denotes its Cholesky factor, which satisfies $\left(Z^{\frac{1}{2}}\right)^{\top} Z^{\frac{1}{2}}=$ $Z^{\frac{1}{2}}\left(Z^{\frac{1}{2}}\right)^{\top}=Z$ and, $\lambda_{\min }(Z)$ and $\lambda_{\max }(Z)$ denote the smallest and the largest eigenvalue of $Z$, respectively.

\section{B. Input-to-state stabilization conditions for min-max MPC}

In this paper we consider the class of constrained discretetime linear systems affected, possibly simultaneously, by parametric uncertainties and additive disturbance inputs, i.e.

$$
x_{k+1}=A\left(w_{k}\right) x_{k}+B\left(w_{k}\right) u_{k}+E\left(w_{k}\right) v_{k}, \quad k \in \mathbb{Z}_{+},
$$

where $x_{k} \in \mathbb{X} \subset \mathbb{R}^{n}$ is the state, $u_{k} \in \mathbb{U} \subset \mathbb{R}^{m}$ is the control action, $w_{k} \in \mathbb{W} \subset \mathbb{R}^{d_{w}}$ is an unknown time-varying parametric uncertainty and $v_{k} \in \mathbb{V} \subset \mathbb{R}^{d_{v}}$ is an unknown time-varying additive disturbance input. The sets $\mathbb{X}$ and $\mathbb{U}$ are assumed to be compact and to contain the origin in their interior, while $\mathbb{W}$ and $\mathbb{V}$ are assumed to be compact polyhedral sets. Here, $A(w) \in \mathbb{R}^{n \times n}, B(w) \in \mathbb{R}^{n \times m}$ and $E(w) \in \mathbb{R}^{n \times d_{v}}$ are assumed to be affine functions of $w \in \mathbb{W}$. We also assume that there exists a $w \in \mathbb{W}$ such that the matrix $B(w)$ has full-column rank.

Next, we consider the case when feedback min-max MPC [1], [8], [9], [13] is employed to calculate the control action $u_{k}, k \in \mathbb{Z}_{+}$, in (1) and we briefly present the corresponding optimization problem set-up. For a fixed $N \in \mathbb{Z}_{\geq 1}$ let $\mathbf{w}_{k}^{M}:=\left(w_{0 \mid k}^{M}, \ldots, w_{N-1 \mid k}^{M}\right)$ and $\mathbf{v}_{k}^{M}:=\left(v_{0 \mid k}^{M}, \ldots, v_{N-1 \mid k}^{M}\right)$ denote all possible future disturbance sequences realizations, where $M \in \mathscr{M}$ ( $\mathscr{M}$ has infinite dimension) indexes the realizations. We define an input scenario at time $k \in \mathbb{Z}_{+}$and for given state $x_{k}$ as a map $\pi: \mathscr{M} \rightarrow \mathbb{U}^{N}$ that assigns to each index $M \in \mathscr{M}$ a sequence of control laws, i.e. $\pi(M):=\mathbf{u}_{k}^{M}:=$ $\left(u_{0 \mid k}^{M}, \ldots, u_{N-1 \mid k}^{M}\right) \in \mathbb{U}^{N}$. A scenario can be interpreted as a policy that specifies for any realization of the disturbance a corresponding control sequence to be applied. Given $x_{k}, k \in$ $\mathbb{Z}_{+}$, we denote by $\mathbf{x}_{k}(M):=\left(x_{1 \mid k}^{M}, \ldots, x_{N \mid k}^{M}\right)$ the corresponding predicted state sequence starting at $x_{0 \mid k}^{M}:=x_{k}, M \in \mathscr{M}$, and where

$$
x_{i \mid k}^{M}:=A\left(w_{i-1 \mid k}^{M}\right) x_{i-1 \mid k}^{M}+B\left(w_{i-1 \mid k}^{M}\right) u_{i-1 \mid k}^{M}+E\left(w_{i-1 \mid k}^{M}\right) v_{i-1 \mid k}^{M}
$$

for all $i=1, \ldots, N$. Furthermore, let $\mathbb{X}_{T} \subseteq \mathbb{X}$ denote a desired target set that contains the origin in its interior. Consider now the following set of admissible input scenarios defined with respect to $\mathbb{X}_{T}$ and state $x_{k} \in \mathbb{X}$ :

$$
\begin{aligned}
& \overline{\mathscr{U}}_{N}\left(x_{k}\right):=\left\{\pi: \mathscr{M} \rightarrow \mathbb{U}^{N} \mid\right. \\
& \quad \mathbf{x}_{k}(M) \in \mathbb{X}^{N}, x_{N \mid k}^{M} \in \mathbb{X}_{T}, \text { for all } M \in \mathscr{M}, \\
& \quad x_{i \mid k}^{M_{1}}=x_{i \mid k}^{M_{2}} \Rightarrow u_{i \mid k}^{M_{1}}=u_{i \mid k}^{M_{2}}, \\
& \left.\quad \text { for all } i=0, \ldots, N-1,\left(M_{1}, M_{2}\right) \in \mathscr{M} \times \mathscr{M}\right\} .
\end{aligned}
$$

Let $F: \mathbb{R}^{n} \rightarrow \mathbb{R}_{+}$with $F(0)=0$ and $L: \mathbb{R}^{n} \times \mathbb{R}^{m} \rightarrow \mathbb{R}_{+}$with $L(0,0)=0$ be continuous, convex and bounded functions. The feedback min-max MPC optimization problem is formulated next.

Problem II.1 Let the target set $\mathbb{X}_{T} \subseteq \mathbb{X}$ and $N \in \mathbb{Z}_{\geq 1}$ be given. At time $k \in \mathbb{Z}_{+}$let $x_{k} \in \mathbb{X}$ be given and minimize

$$
\max _{M \in \mathscr{M}}\left\{F\left(x_{N \mid k}^{M}\right)+\sum_{i=0}^{N-1} L\left(x_{i \mid k}^{M}, u_{i \mid k}^{M}\right)\right\},
$$

with prediction model (1), over all admissible input scenarios $\pi \in \overline{\mathscr{U}}_{N}\left(x_{k}\right)$.

Let $\pi^{*}$ be an optimal scenario (obtained by solving Problem II.1) with $\pi^{*}(M):=\mathbf{u}_{k}^{M *}:=\left(u_{0 \mid k}^{M *}, \ldots, u_{N-1 \mid k}^{M *}\right), M \in \mathscr{M}$. Note that due to the non-anticipative constraints imposed in $\overline{\mathscr{U}}_{N}\left(x_{k}\right)$ it holds that $u_{0 \mid k}^{M_{1} *}=u_{0 \mid k}^{M_{2} *}$ for all $\left(M_{1}, M_{2}\right) \in \mathscr{M} \times \mathscr{M}$.

A state $x \in \mathbb{X}$ is called feasible for Problem II.1 if $\overline{\mathscr{U}}_{N}(x) \neq \emptyset$. Similarly, Problem II.1 is said to be feasible for $x \in \mathbb{X}$ if $\overline{\mathscr{U}}_{N}(x) \neq \emptyset$. Let $\mathbb{X}_{f}(N) \subseteq \mathbb{X}$ denote the set of feasible states with respect to Problem II.1. The feedback min-max MPC control law is obtained as

$$
\bar{u}\left(x_{k}\right):=u_{0 \mid k}^{M *} ; \quad M \in \mathscr{M}, k \in \mathbb{Z}_{+} .
$$

Next, we summarize recently developed [6] a priori sufficient conditions for assuring robust stability in terms of input-to-state practical stability (ISpS) (with respect to the disturbance input $v \in \mathbb{V}$ ) of system (1) in closed-loop with the min-max MPC control law (2) (see also [1], [5], [7] and the references therein). Let $h: \mathbb{R}^{n} \rightarrow \mathbb{R}^{m}$ denote an auxiliary state-feedback control law with $h(0)=0$ and let $\mathbb{X}_{\mathbb{U}}:=\{x \in \mathbb{X} \mid h(x) \in \mathbb{U}\}$.

Assumption II.2 There exist $a, a_{1}, b, \lambda>0$ with $a \leq b$ and a $\mathscr{K}$-function $\sigma(\cdot)$ such that:

1) $\mathbb{X}_{T} \subseteq \mathbb{X}_{\mathbb{U}}$

2) $\mathbb{X}_{T}$ is a Robust Positively Invariant (RPI) set [6] for system (1) in closed-loop with $u_{k}=h\left(x_{k}\right), k \in \mathbb{Z}_{+}$;

3) $L(x, u) \geq a\|x\|^{\lambda}$ for all $x \in \mathbb{X}$ and all $u \in \mathbb{U}$;

4) $a_{1}\|x\|^{\lambda} \leq F(x) \leq b\|x\|^{\lambda}$ for all $x \in \mathbb{X}_{T}$;

5) $F(A(w) x+B(w) h(x)+E(w) v)-F(x) \leq-L(x, h(x))+$ $\sigma(\|v\|)$ for all $x \in \mathbb{X}_{T}, w \in \mathbb{W}$ and all $v \in \mathbb{V}$.

Remark II.3 It can be proven that Assumption II.2 is sufficient for guaranteeing input-to-state stability of the min-max MPC closed-loop system, if the control law $h(\cdot)$ is employed for all states in the terminal set $\mathbb{X}_{T}$. See [6] for details.

Remark II.4 The sufficient conditions of Assumption II.2 can be regarded as an extension to min-max MPC of the 
well known nominal (i.e. in the absence of perturbations) stabilization conditions for MPC [1]. As the stabilization conditions for MPC [1] require that the terminal cost is a local Lyapunov function for the system in closed-loop with an auxiliary state-feedback control law, similarly, Assumption II.2 requires that the terminal cost is a local Input-toState Stable (ISS) Lyapunov function [10] for the system in closed-loop with the auxiliary control law $h(\cdot)$.

Note that the ISpS results based on Assumption II.2 also apply to open-loop min-max MPC schemes, such as the ones presented in [4], [12]. In Section V we will use the min-max MPC algorithm of [12] to illustrate the developed theory.

\section{PROBLEM STATEMENT}

For a given stage cost $L(\cdot, \cdot)$, in order to employ Assumption II.2 for setting-up min-max MPC schemes with an a priori ISpS guarantee, one needs efficient methods for computing a terminal cost $F(\cdot)$, a terminal set $\mathbb{X}_{T}$ and a control law $h(\cdot)$ that satisfy Assumption II.2.

Note that, once $F(\cdot)$ and $h(\cdot)$ are known, several methods are available for calculating the maximal RPI set contained in $\mathbb{X}_{\mathbb{U}}$ for system (1) in closed-loop with $u_{k}=h\left(x_{k}\right), k \in \mathbb{Z}_{+}$, see, for example, [14]-[16].

The problem addressed in this paper is how to calculate $F(\cdot)$ and $h(\cdot)$ such that Assumption II.2 (especially condition Assumption II.2-5)) holds. This comes down to computing an input-to-state stabilizing state-feedback (i.e. $h(\cdot))$ along with an ISS Lyapunov function (i.e. $F(\cdot)$ ) for system (1). This is a non-trivial problem, which depends on the type of MPC cost and hence, on the type of candidate ISS Lyapunov function.

\section{MAIN RESULTS}

In this section we assume that the stage cost $L(\cdot, \cdot)$ is given and we present systematic methods for computing a terminal cost $F(\cdot)$ and a control law $h(\cdot)$ that satisfy Assumption II.2, with special focus on condition Assumption II.2-5).

\section{A. Quadratic MPC Costs}

First, we consider the case when quadratic forms are used to define the MPC cost function, i.e. $F(x):=x^{\top} P x$ and $L(x, u):=x^{\top} Q x+u^{\top} R u$, where $P, Q \in \mathbb{R}^{n \times n}$ and $R \in$ $\mathbb{R}^{m \times m}$ are assumed to be positive definite and symmetric matrices. Then, it follows that $L(x, u) \geq x^{\top} Q x \geq \lambda_{\min }(Q)\|x\|_{2}^{2}$ for all $x \in \mathbb{R}^{n}$ and all $u \in \mathbb{R}^{m}$ and $\lambda_{\min }(P)\|x\|_{2}^{2} \leq F(x) \leq$ $\lambda_{\max }(P)\|x\|_{2}^{2}$ for all $x \in \mathbb{R}^{n}$. Hence, Assumption II.2-3),4) is generically satisfied for $a:=\lambda_{\min }(Q)>0, a_{1}:=\lambda_{\min }(P)>0$, $b:=\lambda_{\max }(P)>0$ and $\lambda=2$.

Next, we present LMI based sufficient conditions for Assumption II.2-5) to be satisfied. Let $Q$ and $R$ be known positive definite and symmetric matrices and let $\tau>0$ be a given number. Consider now the following matrix inequality:

$$
\Delta(w):=\left(\begin{array}{ll}
\Delta_{11} & \Delta_{12} \\
\Delta_{21} & \Delta_{22}
\end{array}\right)>0,
$$

where

$$
\Delta_{11}:=\left(\begin{array}{cc}
Z & -(A(w) Z+B(w) Y)^{\top} \\
-(A(w) Z+B(w) Y) & \tau Z
\end{array}\right),
$$

$$
\begin{aligned}
& \Delta_{22}:=\operatorname{diag}\left(\left[\left(\begin{array}{cc}
Z & 0 \\
0 & Z
\end{array}\right),\left(\begin{array}{cc}
Q^{-1} & 0 \\
0 & Q^{-1}
\end{array}\right),\left(\begin{array}{ccc}
R^{-1} & 0 \\
0 & R^{-1}
\end{array}\right)\right]\right), \\
& \Delta_{12}=\Delta_{21}^{\top}:=\left(\begin{array}{cccccc}
(A(w) Z+B(w) Y)^{\top} & 0 & Z & 0 & Y^{\top} & 0 \\
0 & 0 & 0 & 0 & 0 & 0
\end{array}\right) .
\end{aligned}
$$

In (3), the operator $\operatorname{diag}\left(\left[Z_{1}, \ldots, Z_{n}\right]\right)$ denotes a diagonal matrix of appropriate dimensions with the matrices $Z_{1}, \ldots, Z_{n}$ on the main diagonal, and 0 denotes a zero matrix of appropriate dimensions. The unknown variables in the matrix inequality (3) are the matrix $Z \in \mathbb{R}^{n \times n}$, which is required to be positive definite and symmetric, and the matrix $Y \in \mathbb{R}^{m \times n}$. Notice that (3) is an LMI for a fixed value of $w$.

\section{Theorem IV.1 Suppose that the LMIs}

$$
\Delta\left(w^{s}\right)>0, \quad s \in \mathscr{S},
$$

in the unknowns $Z, Y$ are feasible, where $\left\{w^{s} \mid s \in \mathscr{S}=\right.$ $\{1, \ldots, S\}\}$ are the vertices of $\mathbb{W}$. Let $Z>0$ and $Y$ be a solution of (4). Then, the matrix $P:=Z^{-1}$ and the control law $h(x):=K x$ with the feedback gain $K:=Y Z^{-1}$ satisfy Assumption II.2-5) for all $x \in \mathbb{R}^{n}, w \in \mathbb{W}$ and all $v \in \mathbb{R}^{d_{v}}$, and with $\sigma(\|v\|):=(1+\tau)\left\|P^{\frac{1}{2}}\right\|_{2}^{2} \max _{w \in \mathbb{W}}\|E(w)\|_{2}^{2}\|v\|_{2}^{2}$.

Proof: Since $\left\{w^{s} \mid s \in \mathscr{S}=\{1, \ldots, S\}\right\}$ are the vertices of the polyhedron $\mathbb{W}$ and $A(w), B(w)$ are affine functions of $w$, the matrix inequality (3) is convex in $w$. Therefore, if (4) holds, it follows that (3) holds, i.e.

$$
\Delta(w)>0, \quad \forall w \in \mathbb{W} .
$$

Then, applying the Schur complement to (3) we obtain

$$
\Delta_{11}-\Delta_{12} \Delta_{22}^{-1} \Delta_{21}>0, \quad \forall w \in \mathbb{W},
$$

which yields the equivalent matrix inequality:

$\Theta_{Z}(w):=\left(\begin{array}{cc}\Xi_{Z}(w) & -(A(w) Z+B(w) Y)^{\top} \\ -(A(w) Z+B(w) Y) & \tau Z\end{array}\right)>0$

for all $w \in \mathbb{W}$, where

$$
\begin{aligned}
\Xi_{Z}(w):= & Z-(A(w) Z+B(w) Y)^{\top} Z^{-1}(A(w) Z+B(w) Y) \\
& -Z Q Z-Y^{\top} R Y .
\end{aligned}
$$

Next, by pre and post-multiplying the matrix inequality $\Theta_{Z}(w)>0$ with the positive definite matrix $\left(\begin{array}{cc}Z^{-1} & 0 \\ 0 & Z^{-1}\end{array}\right)$ and by replacing $Z^{-1}$ with $P$ and $Y Z^{-1}$ with $K$ in the resulting matrix inequality we obtain:

$\Theta_{P}(w):=\left(\begin{array}{cc}\Xi_{P}(w) & -(A(w)+B(w) K)^{\top} P \\ -P(A(w)+B(w) K) & \tau P\end{array}\right)>0$

for all $w \in \mathbb{W}$, where

$$
\begin{aligned}
\Xi_{P}(w):= & P-(A(w)+B(w) K)^{\top} P(A(w)+B(w) K) \\
& -Q-K^{\top} R K .
\end{aligned}
$$

This implies that

$$
\left[\begin{array}{ll}
x^{\top} & v^{\top} E(w)^{\top}
\end{array}\right] \Theta_{P}(w)\left[\begin{array}{c}
x \\
E(w) v
\end{array}\right] \geq 0,
$$


for all $x \in \mathbb{R}^{n}, w \in \mathbb{W}$ and all $v \in \mathbb{R}^{d_{v}}$, which yields:

$$
\begin{aligned}
& x^{\top} \Xi_{P}(w) x-v^{\top} E(w)^{\top} P(A(w)+B(w) K) x \\
& -x^{\top}(A(w)+B(w) K)^{\top} P E(w) v-v^{\top} E(w)^{\top} P E(w) v \\
& +(1+\tau) v^{\top} E(w)^{\top} P E(w) v \geq 0 \Leftrightarrow x^{\top} P x-x^{\top} Q x-x^{\top} K^{\top} R K x \\
& -((A(w)+B(w) K) x+E(w) v)^{\top} P((A(w)+B(w) K) x+E(w) v) \\
& +(1+\tau) v^{\top} E(w)^{\top} P E(w) v \geq 0 .
\end{aligned}
$$

Note that in (6) we added and subtracted the term $v^{\top} E(w)^{\top} P E(w) v$. Next, consider the following function:

$$
\bar{\sigma}(w, v):=(1+\tau) v^{\top} E(w)^{\top} P E(w) v=(1+\tau)\left\|P^{\frac{1}{2}} E(w) v\right\|_{2}^{2},
$$

and choose $\sigma(\|v\|):=(1+\tau)\left\|P^{\frac{1}{2}}\right\|_{2}^{2} \max _{w \in \mathbb{W}}\|E(w)\|_{2}^{2}\|v\|_{2}^{2}$. Using the fact that $\bar{\sigma}(w, v) \leq \sigma(\|v\|)$ for all $w \in \mathbb{W}$ and all $v \in \mathbb{R}^{d_{v}}$, inequality (6), and letting $h(x):=K x$ we obtain:

$$
F(A(w) x+B(w) h(x)+E(w) v)-F(x) \leq-L(x, h(x))+\sigma(\|v\|) .
$$

Hence, Assumption II.2-5) is satisfied for all $x \in \mathbb{R}^{n}, w \in \mathbb{W}$ and all $v \in \mathbb{R}^{d_{v}}$, and with

$$
\sigma(\|v\|):=(1+\tau)\left\|P^{\frac{1}{2}}\right\|_{2}^{2} \max _{w \in \mathbb{W}}\|E(w)\|_{2}^{2}\|v\|_{2}^{2} .
$$

The only thing left to prove is that $\lambda_{\min }(Q)=: a \leq b:=$ $\lambda_{\max }(P)$. As shown above, for all $w \in \mathbb{W}$ it holds that:

$$
\Theta_{P}(w)>0 \Rightarrow \Xi_{P}(w)>0 \Rightarrow P-Q>0 \Rightarrow x^{\top}(P-Q) x \geq 0,
$$

for all $x \in \mathbb{R}^{n}$. Then, it follows that $a\|x\|_{2}^{2} \leq x^{\top} Q x \leq x^{\top} P x \leq$ $b\|x\|_{2}^{2}$, for all $x \in \mathbb{R}^{n}$ and therefore, $a \leq b$.

The number $\tau>0$ and the matrix $P$ yield the gain of the $\mathscr{K}$-function $\sigma(\cdot)$, which is related to the robust performance of the closed-loop system (see [6] for details). To optimize over this gain, $\tau$ can also be defined as an unknown variable in (4), which results in a bilinear matrix inequality (i.e. due to the term $\tau P$ ). However, since the unknown $\tau$ is a scalar, this problem can be solved efficiently via semi-definite programming solvers [17], [18] by setting lower and upper bounds for $\tau$ and doing bisections.

Remark IV.2 The solution provided by Theorem IV.1 implies that condition Assumption II.2-5) holds for all $x$ and all $v$. This introduces some conservativeness, as condition Assumption II.2-5) only needs to be satisfied for $x \in \mathbb{X}_{T}$ and $v \in \mathbb{V}$. However, note that the set $\mathbb{X}_{T}$ depends on the feedback $K$ and therefore, it cannot be specified before finding a solution that satisfies condition Assumption II.2-5). This is also the case for nominally stabilizing MPC, where the condition corresponding to Assumption II.2-5) is solved for all $x$, see [1]. With respect to additive disturbances, an attempt to solve Assumption II.2-5) only for $v \in \mathbb{V}$ was made in [4]. Unfortunately, this approach led to a bilinear matrix inequality in the matrices $P$ and $K$, which cannot be solved in a systematic way. From this point of view, the solution of Theorem IV.1 is less conservative because it offers a computationally efficient set-up for simultaneously finding both $P$ and $K$.
Remark IV.3 The computation of an input-to-state stabilizing state-feedback for the linear system (1) can be related to the discrete-time $\mathscr{H}_{\infty}$ synthesis problem. However, the well known LMI based solution to the discrete-time $\mathscr{H}_{\infty}$ synthesis problem, see, for example, [19] and the references therein, cannot be used to obtain a solution to Assumption II.2-5). This is due to the additional term $x^{\top} K^{\top} R K x$ in the unknown $K$ introduced by the MPC stage cost. If one employs the $\mathscr{H}_{\infty}$ synthesis approach (see [19], Section 2.1) to solve Assumption II.2-5), one obtains a bilinear matrix inequality in the matrices $P$ and $K$, which is difficult to solve.

\section{B. 1-norms or $\infty$-norms MPC Costs}

Now we consider the case when 1 -norms or $\infty$-norms are used to define the MPC cost function, i.e. $F(x):=\|P x\|$ and $L(x, u):=\|Q x\|+\|R u\|$, where $P \in \mathbb{R}^{n_{p} \times n}, Q \in \mathbb{R}^{n_{q} \times n}$ and $R \in \mathbb{R}^{m_{r} \times m}$ are assumed to be matrices that have full-column rank and $\|\cdot\|$ denotes either the 1 -norm or the $\infty$-norm. For any full-column rank matrix $Z \in \mathbb{R}^{n_{z} \times n}$ there exist $\zeta_{2}(Z) \geq$ $\zeta_{1}(Z)>0$ such that

$$
\zeta_{1}(Z)\|x\| \leq\|Z x\| \leq \zeta_{2}(Z)\|x\|, \quad \forall x \in \mathbb{R}^{n} .
$$

For example, $\zeta_{1}(Z)=\frac{s_{z}}{\sqrt{n_{z}}}$, where $s_{z}>0$ is the smallest singular value of $Z$, and $\zeta_{2}(Z)=\|Z\|$. Then, we have that $L(x, u) \geq\|Q x\| \geq \zeta_{1}(Q)\|x\|$ for all $x \in \mathbb{R}^{n}$ and all $u \in \mathbb{R}^{m}$ and $\zeta_{1}(P)\|x\| \leq F(x) \leq \zeta_{2}(P)\|x\|$ for all $x \in \mathbb{R}^{n}$. Hence, Assumption II.2-3),4) is generically satisfied for $a:=\zeta_{1}(Q)>0$, $a_{1}:=\zeta_{1}(P)>0, b:=\zeta_{2}(P)>0$ and $\lambda=1$.

Next, we present norm inequalities based sufficient conditions for Assumption II.2-5) to be satisfied. Let $Q$ and $R$ be known full-column rank matrices and consider the following norm inequalities:

$$
\begin{aligned}
& 1-\left\|P\left(A\left(w^{s}\right)+B\left(w^{s}\right) K\right) P^{-L}\right\|-\left\|Q P^{-L}\right\|-\left\|R K P^{-L}\right\| \geq 0 \\
& \zeta-\left\|P\left(A\left(w^{s}\right)+B\left(w^{s}\right) K\right)\right\|-\|Q\|-\|R K\| \geq 0,
\end{aligned}
$$

where $P^{-L}=\left(P^{\top} P\right)^{-1} P^{\top}, \zeta>0$ is a fixed number and $\left\{w^{s}\right.$ $s \in \mathscr{S}=\{1, \ldots, S\}\}$ are the vertices of $\mathbb{W}$. The unknown variables in (7) are the matrices $P$ and $K$.

Theorem IV.4 Consider the following hypotheses:

(i) There exist a full-column rank matrix $P$ and a feedback gain $K$ that satisfy inequality (7a) for all $s \in \mathscr{S}$;

(ii) There exist a matrix $P$ and a feedback gain $K$ that satisfy inequality (7b) for all $s \in \mathscr{S}$ and $\|P x\| \geq \zeta\|x\|$ for all $x \in \mathbb{R}^{n}$.

Suppose that either hypothesis (i) or hypothesis (ii) holds. Then, the matrix $P$ and the feedback gain $K$ satisfy Assumption II.2-5) for all $x \in \mathbb{R}^{n}, w \in \mathbb{W}$ and all $v \in \mathbb{R}^{d_{v}}$ and with $\sigma(\|v\|):=\tau\|P\| \max _{w \in \mathbb{W}}\|E(w)\|\|v\|$ for any $\tau \geq 1$.

Proof: (i) Since $\left\{w^{s} \mid s \in \mathscr{S}=\{1, \ldots, S\}\right\}$ are the vertices of the polyhedron $\mathbb{W}$ and $A(w), B(w)$ are affine functions of $w$, it follows that inequality (7a) is convex in $w$. Therefore, from the hypothesis (i), we obtain that:

$$
1-\left\|P(A(w)+B(w) K) P^{-L}\right\|-\left\|Q P^{-L}\right\|-\left\|R K P^{-L}\right\| \geq 0,
$$


for all $w \in \mathbb{W}$. Then, by post-multiplying (8) with $\|P x\|>0$ and using the triangle inequality yields:

$$
\begin{aligned}
0 & \leq\|P x\|-\left\|P(A(w)+B(w) K) P^{-L}\right\|\|P x\|-\left\|Q P^{-L}\right\|\|P x\| \\
& -\left\|R K P^{-L}\right\|\|P x\| \leq\|P x\|-\left\|P(A(w)+B(w) K) P^{-L} P x\right\| \\
& -\left\|Q P^{-L} P x\right\|-\left\|R K P^{-L} P x\right\| \leq\|P x\|-\|Q x\|-\|R K x\| \\
& -\|P(A(w)+B(w) K) x\|+(\tau-1)\|P E(w) v\| \\
& \leq\|P x\|-\|P((A(w)+B(w) K) x+E(w) v)\|-\|Q x\| \\
& -\|R K x\|+\tau\|P E(w) v\|, \quad \forall \tau \geq 1 .
\end{aligned}
$$

Letting $h(x):=K x$ and $\sigma(\|v\|):=\tau\|P\| \max _{w \in \mathbb{W}}\|E(w)\|\|v\|$ we obtain

$$
F(A(w) x+B(w) h(x)+E(w) v)-F(x) \leq-L(x, h(x))+\sigma(\|v\|) .
$$

Hence, Assumption II.2-5) is satisfied for all $x \in \mathbb{R}^{n}, w \in \mathbb{W}$ and all $v \in \mathbb{R}^{d_{v}}$ and with $\sigma(\|v\|):=\tau\|P\| \max _{w \in \mathbb{W}}\|E(w)\|\|v\|$ for any $\tau \geq 1$. Finally, from the above inequality we obtain:

$$
\zeta_{1}(Q)\|x\| \leq\|Q x\| \leq\|P x\| \leq \zeta_{2}(P)\|x\|, \quad \forall x \in \mathbb{R}^{n},
$$

and therefore, $\zeta_{1}(Q)=: a \leq b:=\zeta_{2}(P)$.

(ii) The proof is obtained analogously to the proof of (i), by post-multiplying the inequality obtained from (7b) as in (8) with $\|x\|$ and using $\|P x\| \geq \zeta\|x\|$ for all $x \in \mathbb{R}^{n}$.

A solution that satisfies the norm inequalities (7) can be obtained by minimizing

$$
\begin{aligned}
& J_{1}(P, K):= \\
& \max _{s \in \mathscr{S}}\left\{\left\|P\left(A\left(w^{S}\right)+B\left(w^{S}\right) K\right) P^{-L}\right\|+\left\|Q P^{-L}\right\|+\left\|R K P^{-L}\right\|\right\},
\end{aligned}
$$

if the resulting value function is less than 1 ; and by minimizing

$$
J_{2}(P, K):=\max _{s \in \mathscr{S}}\left\{\left\|P\left(A\left(w^{s}\right)+B\left(w^{s}\right) K\right)\right\|+\|Q\|+\|R K\|\right\},
$$

if the resulting value function is less than $\zeta$, and $\|P x\| \geq$ $\zeta\|x\|$. These are non-convex nonlinear optimization problems, which can be solved using black-box optimization solvers, such as the fmincon Matlab solver. To ensure that the matrix $P$ has full-column rank one can impose the nonlinear constraint $\operatorname{det}\left(P^{\top} P\right)>0$ using fmincon. Alternatively, one can directly specify the condition $J_{1}(P, K) \leq 1\left(J_{2}(P, K) \leq \zeta\right)$ as a nonlinear constraint using fmincon.

The optimization problems associated to (7a) and (7b) can be simplified if one of the unknowns is fixed using an educated guess. For example, a locally stabilizing feedback $K$ can be calculated via the LMI approach of Theorem IV.1. Then, fixing $K$ in (7b) and solving in $P$ amounts to searching for a piecewise linear Lyapunov function for a system which already admits a quadratic Lyapunov function, which is not conservative. Alternatively, $P$ can be chosen as the matrix that defines a 0 -symmetric polyhedral RPI set for system (1) in closed-loop with $h(\cdot)$, e.g. obtained using the MPT [16]. Then, fixing $P$ in (7a) or (7b) and solving in $K$ boils down to searching for a different feedback which renders the polyhedral set induced by $F(\cdot)$ RPI and, moreover, is such that (7a) or (7b), respectively, holds.
Remark IV.5 The optimization problems for computing a terminal cost formulated in this section, for both quadratic and 1-norm or $\infty$-norm costs, do not depend on the dimension $d_{v}$ of the additive disturbance vector, or on the number of vertices of the set $\mathbb{V}$. Furthermore, the number of LMIs or norm inequalities it is always porportional to the number of vertices of the set $\mathbb{W}$. However, the number of optimization variables remains the same, irrespective of the number of vertices of the sets $\mathbb{V}$ and $\mathbb{W}$.

\section{AN ACTIVE SUSPENSION SYSTEM}

Consider the problem of robustly regulating to the origin the following active suspension system [2], [20]:

$$
\begin{aligned}
x_{k+1}= & {\left[\begin{array}{cccc}
0.809 & 0.009 & 0 & 0 \\
-36.93 & 0.80 & 0 & 0 \\
0.191 & -0.009 & 1 & 0.01 \\
0 & 0 & 0 & 1
\end{array}\right] x_{k} } \\
& +\left[\begin{array}{c}
0.0005 \\
0.0935 \\
-0.005 \\
-0.01
\end{array}\right] u_{k}+\left[\begin{array}{c}
-0.009 \\
0.191 \\
-0.0006 \\
0
\end{array}\right] v_{k}, \quad k \in \mathbb{Z}_{+},
\end{aligned}
$$

where the disturbance input $v_{k}$ represents the vertical ground velocity of the road profile and the control action $u_{k}$ represents the vertical acceleration. The additive disturbance $v_{k}$ takes values in the set $\mathbb{V}:=\{v \in \mathbb{R} \mid-0.4 \leq v \leq 0.4\}$, for all $k \in \mathbb{Z}_{+}$. The first and the third state are constrained between -0.02 and 0.02 , and -0.05 and 0.05 , respectively. The control action is constrained between -5 and 5 .

To ensure input-to-state practical stability, we employed the LMI approach of Theorem IV.1 for computing a quadratic forms based terminal cost and a local state-feedback controller that satisfy Assumption II.2. The following terminal weight $P$ and feedback $K$ were obtained for the same stage cost weights used in [2], i.e. $Q=\operatorname{diag}([5000,0.1,400,0.1])$, $R=1.8$, and for $\tau=100$ :

$$
\begin{gathered}
P=10^{4}\left[\begin{array}{cccc}
7.5651 & 0.0079 & 0.3702 & -0.0156 \\
0.0079 & 0.0017 & 0.0001 & 0.0001 \\
0.3702 & 0.0001 & 0.7241 & 0.0250 \\
-0.0156 & 0.0001 & 0.0250 & 0.0303
\end{array}\right], \\
K=\left[\begin{array}{llll}
20.7682 & -0.8657 & 16.8717 & 2.1688
\end{array}\right]
\end{gathered}
$$

The terminal constraint set $\mathbb{X}_{T}$ (a polyhedron with 56 vertices, see Figure 1 for a 3D section plot) was calculated as the maximal RPI set inside $\mathbb{X}_{\mathbb{U}}$ for system (9) in closed-loop with $h(x)=K x$ using the MPT [16]. The evolution in time of the states, additive disturbance input and min-max MPC control action is plotted in Figure 2. The open-loop minmax MPC control action was calculated for $N=6$ using the numerical set-up of [12] to obtain an explicit solution to the min-max MPC optimization problem. As it can be observed in Figure 2, the closed-loop system is robustly stable and the state converges to the origin when the additive disturbance vanishes. 


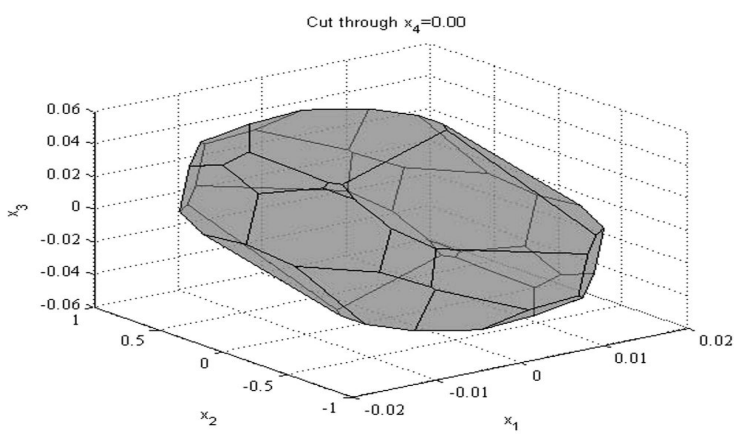

Fig. 1. 3D section of the terminal constraint set $\mathbb{X}_{T}$.

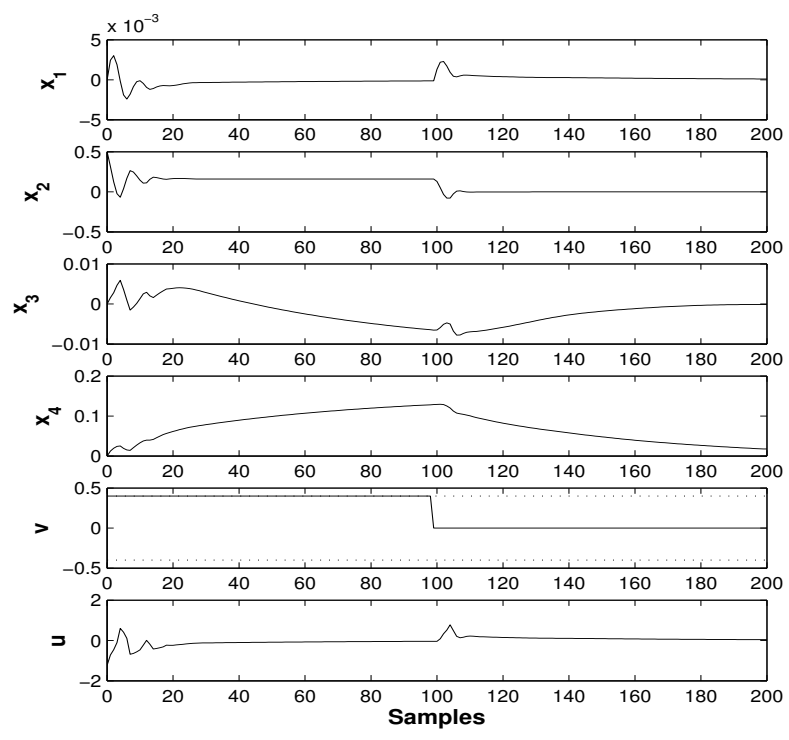

Fig. 2. States, additive disturbance input and control action histories.

\section{CONCLUSIONS}

In this paper we presented new techniques for computing a terminal cost and a local state-feedback controller that satisfy input-to-state stabilization conditions for min-max MPC. Min-max MPC controllers based on both quadratic and 1-norms or $\infty$-norms costs were considered. Compared to existing approaches, the proposed techniques can be applied to linear systems affected simultaneously by timevarying parametric uncertainties and additive disturbances. The resulting MPC cost function is continuous, convex and bounded, which is desirable from an optimization point of view. The proposed techniques employ linear matrix inequalities in the case of quadratic MPC cost functions and norm inequalities in the case of MPC cost functions defined using 1-norms or $\infty$-norms. The effectiveness of the developed computational methods was illustrated by means of a simulated active suspension system.

\section{ACKNOWLEDGMENTS}

This research was supported by the Dutch Science Foundation (STW), Grant "Model Predictive Control for Hybrid
Systems" (DMR. 5675), the European Community through the Network of Excellence HYCON (contract FP6-IST511368) and the Spanish MEC (DPI 2005-04568).

\section{REFERENCES}

[1] D. Q. Mayne, J. B. Rawlings, C. V. Rao, and P. O. M. Scokaert, "Constrained model predictive control: Stability and optimality," $A u$ tomatica, vol. 36, pp. 789-814, 2000.

[2] A. Bemporad, F. Borrelli, and M. Morari, "Min-max control of constrained uncertain discrete-time linear systems," IEEE Transactions on Automatic Control, vol. 48, no. 9, pp. 1600-1606, 2003.

[3] B. Pluymers, J. A. Rossiter, J. A. K. Suykens, and B. De Moor, "Interpolation based MPC for LPV systems using polyhedral invariant sets," in American Control Conference, Portland, Oregon, 2005, pp. $810-815$.

[4] T. Alamo, D. Muñoz de la Peña, D. Limon, and E. F. Camacho, "Constrained min-max predictive control: Modifications of the objective function leading to polynomial complexity," IEEE Transactions on Automatic Control, vol. 50, no. 5, pp. 710-714, 2005.

[5] D. Limon, T. Alamo, F. Salas, and E. F. Camacho, "Input to state stability of min-max MPC controllers for nonlinear systems with bounded uncertainties," Automatica, vol. 42, pp. 797-803, 2006.

[6] M. Lazar, D. Muñoz de la Peña, W. P. M. H. Heemels, and T. Alamo, "Min-max nonlinear model predictive control with guaranteed inputto-state stability," in 17th Symposium on Mathematical Theory for Networks and Systems, Kyoto, Japan, 2006.

[7] L. Magni, D. M. Raimondo, and R. Scattolini, "Regional input-to-state stability for nonlinear model predictive control," IEEE Transactions on Automatic Control, vol. 51, no. 9, pp. 1548-1553, 2006.

[8] P. O. M. Scokaert and D. Q. Mayne, "Min-max feedback model predictive control for constrained linear systems," IEEE Transactions on Automatic Control, vol. 43, no. 8, pp. 1136-1142, 1998.

[9] M. V. Kothare, V. Balakrishnan, and M. Morari, "Robust constrained model predictive control using linear matrix inequalities," Automatica, vol. 32, no. 10, pp. 1361-1379, 1996.

[10] Z.-P. Jiang and Y. Wang, "Input-to-state stability for discrete-time nonlinear systems," Automatica, vol. 37, pp. 857-869, 2001.

[11] D. Muñoz de la Peña, T. Alamo, and A. Bemporad, "A decomposition algorithm for feedback min-max model predictive control," IEEE Transactions on Automatic Control, vol. 51, no. 10, pp. 1688-1692, 2006.

[12] D. Muñoz de la Peña, T. Alamo, D. R. Ramirez, and E. F. Camacho, "Min-max model predictive control as a quadratic program," accepted for publication in IEE Proceedings on Control Theory \& Applications.

[13] J. H. Lee and Z. Yu, "Worst-case formulations of model predictive control for systems with bounded parameters," Automatica, vol. 33, no. 5 , pp. 763-781, 1997.

[14] E. M. Gilbert and K. T. Tan, "Linear systems with state and control constraints: The theory and application of maximal output admissible sets," IEEE Transactions on Automatic Control, vol. 36, no. 9, pp. 1008-1020, 1991.

[15] I. Kolmanovsky and E. G. Gilbert, "Theory and computation of disturbance invariant sets for discrete-time linear systems," Mathematical Problems in Engineering, vol. 4, pp. 317-367, 1998.

[16] M. Kvasnica, P. Grieder, M. Baotic, and M. Morari, "Multi Parametric Toolbox (MPT)," in Hybrid Systems: Computation and Control, ser. Lecture Notes in Computer Science, Volume 2993. Pennsylvania, Philadelphia, USA: Springer Verlag, Mar. 2004, pp. 448-462, http://control.ee.ethz.ch/ mpt.

[17] J. Löfberg, "YALMIP: Matlab toolbox for rapid prototyping of optimization problems," 2002, web: http://control.ee.ethz.ch/ joloef/yalmip. msql.

[18] J. F. Sturm, "SeDuMi: Matlab toolbox for solving optimization problems over symmetric cones," 2001, toolbox available online at: http://sedumi.mcmaster.ca/.

[19] H. Chen and C. W. Scherer, "Moving horizon $\mathscr{H}_{\infty}$ control with performance adaptation for constrained linear systems," Automatica, vol. 42, no. 6, pp. 1033-1040, 2006.

[20] D. Hrovat, "Survey on advanced suspension developments and related optimal control applications," Automatica, vol. 33, pp. 1781-1817, 1997. 